\title{
Research of Animal Disease Information System Based on GIS Technology
}

\author{
Hongbin Wang*, Lin Li, Jing Dong, Danning Xu, and Jing Li \\ College of Veterinary Medicine, NorthEast Agricultural University, \\ Harbin,Heilongjiang Province, P.R. China, 150030, \\ Tel.: +86-451-55191940; Fax: +86-451-55190470 \\ neau1940@yahoo.com.cn
}

\begin{abstract}
It is necessary to build animal disease information system in order to dynamically monitor and predict diseases distribution, and propagation, such as HPAI and FMD taking place, Powerful functions of data management analysis and display with the GIS can be applied oriented to animal disease information. This disease information platform can be applied to dynamiclly monitor and predict disease, as well as to support the disease prevention decision making, in order to decrease the loss.
\end{abstract}

Keywords: animal disease; GIS; information system.

\section{Introduction}

In recent years, HPAI and FMD diseases as well as other kind of animal diseases have caused disastrous result by disease outbreak and propagation in some areas of China, . With HPAI for example, in 2005 there were 13 areas having avian influenza outbreak. More than 22 million live birds were slaughtered, while 7 persons were infected in China with bird flu, including five deaths. These are seriously hampered the development of livestock and poultry farming. As a result, it is most important to construct an animal disease information system for dynamic monitoring disease distribution and spread. Animal medicine is a subject with multi-branches involving micro-structure as well as macro-system, of which a great amount of data in relation to the macro system are blessed with the features of spatial distribution. It is evident that the occurrence and epidemic of infectious diseases, the distribution and etiology of endemic, the frequently-occurring disease with regional feature, and the animal husbandry and veterinary health institutions are all closely related to the distribution of spatial information Animal disease data relating to the spatial feature is the prerequisite condition for the application of GIS (Jiang Chenghua, 2004).

GIS (Geographic Information System) is a computer aided spatial data information management system. Generally speaking, the application of GIS enables the spatial data management (collection, storage maintenance), implements the analysis (statistics spatial modeling) and graphics display (figures and maps) (Xu yong, 2006). GIS

\footnotetext{
${ }^{*}$ Corresponding author.
} 
integrate the unique map visual effect with the geographical analysis and database operation. It is therefore the important means to realize the decision support management system (Norstrom M, 2001). In the field of animal hygiene, many state agricultural departments are aware of animal hygiene database and the importance of the construction of GIS in order to display, summary and analyze data of animal's epidemic situation. For instance, in 1996 USA established, the "State Animal Health Report System", at the same time Canada, Australia, New Zealand, Thailand, Malaysia, and other countries have operated respectively the similar system with perfective functions. Our Government has also awared of this requirement, and, established the National Animal Epidemiology Research Center in 2000(Teng Xiangyan, 2005). With a special fund, a national animal health information system has been established integrating health animal health information management and animal health GIS. However in the provinces and cities of our country the livestock diseases geographic information system is hardly established and put into application. Domestic and international GIS application in health research indicates that the technology animal diseases geographic information system based on GIS is an inevitable trend for animal disease information management with scientific and automation feature(Pferiffer D U,2002).

\section{Materials and Methods}

\subsection{System Target}

(1)Integrating correlated data information about livestock and poultry, establish a multi-source databases covering whole province which includes the data of graph,, the data of image and the data of statistic.

(2)Establish and develop a livestock epidemic monitoring information system according with the state standards, and applying GIS technology with web technology. Provide technical support for the construction of provincial units with no provisions for animal epidemic.

(3)Provide the multi-level information service about related information to the distribution, occurrence and prediction of disease for the provincial animal husbandry, veterinary department, vet workers and farmers.

(4)Provide decision support for the provincial animal husbandry and veterinary department.

\subsection{System Framework and Basic Functions}

\section{(1)GIS platform}

The GIS support platform is established with ESRI company's groupware GIS package Mapobjccts (hereinafter referred to as the MO). The application system is an user-oriented program by Visual Basic language 6 , based on the Windows operating system.

(2) The spatial database and attribute database drawing the corresponding thematic maps by using GIS, based on provincial 1:1 million electronic map, and the spatial database and attribute database which established by the obtained epidemic monitoring information. The information of spatial database digital marks according to existing standard map. Including the foundation geographical data such as administrative 
divisions, highway, railway, rivers, lakes and special maps such as animal population spatial distribution, nursery geographical distribution, space scatter diagram after animal epidemics occurrence, the space distribution of transmission intermediary, etc. Attribute database, including soil water, traffic condition, the density of livestock and avian, genius morbi, epidemic disease characteristics, the size of veterinary hospital, etc. Data is available for management by Access database which may link through the SQL.

\section{(3)System structure}

Animal diseases information system diagram is shown in figure 1

System is divided into two major modules: daily management module and decision support module, and divided into three subsystems, and each has its characteristics and functions.

Disease monitoring and management subsystems: which can deal with databases, such as natural resources, animal resource information database, disease database, and make them visualization, scientific, process optimization.

Diseases of comprehensive evaluation subsystems: determine the optimal breeding structure and the breeding density, enact reasonable prevention and control strategies and scheme in key prevention and control areas, through the space analysis and space statistics of relevant data of animal diseases.

Emergency decision of Epidemic outbreak subsystem: scientific and reasonable take emergency measures, reduce the cost, improve the decision-making response speed and accuracy by epidemic situation, GIS technology and many analysis method.

(4)Introduction of functions of the system

(1) Realizing data acquisition, comprehensive inquiry, statistics analysis, and visualization of management

Visual management of livestock and poultry resources, disease spatial databases and attribute data, visualization with epidemic areas data and the reports: information of animal epidemic disease and no provisions for animal epidemic (e.g., husbandry statistical data: veterinary data, immune data etc.)When the map clicked by the attributes tools, this area highlighted and a new form showed with all relevant information of livestock and avian. The data can be printed and forming statements.

Be involved in the management of information and data types and format, data security, data transfer, statements statistics and infuse information system, through the Internet, realize the real-time data acquisition and the outbreak of information flow of information transmission, epidemic disease timely pass the text and images site information, and can be distributed laohutai monitoring and information processing the livestock and poultry. Through the management and livestock data report, realizing the function of data input and report to ensure accurate data and corresponding relationship.

Related information of the livestock can be comprehensive inquired. When the searching information of livestock finished, the results will come from some area that the number of livestock is bigger than a certain data. These areas are presented on map by highlight, this is also applicable to the animal diseases number query a value greater than the query. Make the special map which about total incidence of animals, total loss of animals by the reports of any period of epidemic disease. The information 
of livestock such as variety distribution, personnel distribution, but also provides the technical support for future outbreaks decryption release.

(2) System management of animal husbandry related laws and beforehand library, provide reference for instituting policies and emergency decision-making

Collect the information that about the relevant laws, regulations and standards of animal sanitation which issued of all levels Chinese governments and the relevant departments since 1949, andand various laws and regulations issued of international organizations and other governments. Computerized management of the information systematically and comprehensively. Provide reference for instituting policies.

(3) Analyze the resource of livestock and avian and the space-time information of disease. Provides the basis for livestock production, macro-control of disease control and prevention.

By using spatial analysis function of the GIS (stack analysis, density analysis, cluster analysis, and the dynamic analysis), the human resources and the distribution of resources for livestock and avian can be overall assessment reported, predict distribution of disease for the whole animal diseases regional according to the disease data has been collected and report. Determine the scope of the services by the distance factors, consider the position of existing hygienic medical institutions and radius of services for a variety of factors, and allocate the limited health resources reasonable. Considering the geographical environment, the distribution of existing institution, potential distribution of requirement and other factors, select location scientific by superposition, density and reclassification and other analysis method, provide evidence for selecting the address of new breeding base and veterinarian institutions, etc.

(4) Realizing sudden outbreak positioning, diffusion trend visual simulation, urgent resources optimization ration

Obtaining the information of epidemic-stricken area promptly, accurately, exhaustively confirming livestock epidemic areas it can be divided into several levels showing visual effect of the epidemic area the rational resources and personnel deployment through the hierarchy of epidemic allocated more accurately rational allocating resources

Search the adjoin area of epidemic disease spot Simulate the spread trend of the epidemic disease It may indicate Anti-epidemic station surrounding areas well prepared for prevention and quarantine and the spread of disease Simulate the spread trend of the epidemic disease by some factors such as the choice of livestock epidemic disease in quantity make for prophylaxis

Provide decision support to control epidemic disease by rationing the urgent resources optimally and analyzing epidemic resource information provide scientific control measures for occurred the disease Calculate the distance from the genesis of disease to the epidemic prevention station and provides the optimal path to the epidemic area of infectious disease by network analysis method. Through calculation of the distance, Estimated that veterinary officers to the time the disease occurred, A timely manner in order to control the epidemic. Select the optimal route to the destination, saving the emergency cost.

\section{Summary}

Concerning with the development of livestock influenced seriously by the major disease, poultry aquaculture, this research work has implemented dynamic monitoring 
for livestock diseases distribution and spread based on GIS animal diseases information system,, applying GIS spatial analysis function with network technology..

By combining the GIS technology with network technology, the telemetry data, and the management information from municipal (county) stations are integrated. It is therefore able to monitor the livestock epidemic disease, and to supervise epidemicprevention, and livestock supervisory information in a regular and systematic statistical base. It is also able to grasp the dynamic trend and make data resources visualization. The historical disease condition, spatial distribution rule, etiological analysis of ecological environment, the prevention of disease and reasonable allocation of resources functions may then be analyzed by synthesis. Through the analysis of the deeply used information, the macro management and scientifically decisions of livestock diseases is strengthened. The government administration departments, various monitoring institutions, and scientific research units understand livestock diseases more deeply, and start livestock diseases prevention work with scientific basis.

This system not only provides technology for an animal disease management for the provincial animal husbandry and veterinary department, but also provides good application and technical reference for construction of our animal diseases geographic information system.

\section{Acknowledgements}

The authors thank the financial support from National Project of Scientific and Technical Supporting of China during the 11th Five-year Plan 2006BAD10A02-04.

\section{References}

Chenghua, J., Yang, D.: The application of geography in medicine geography research. Overseas medicine geography fascicl 25(4), 182-184 (2004)

Yong, X., Siqing, Z., Yang, Y.: Research and application of geographic information systems for Prophylaxis and control disease. Disease monitor 21(1), 45-47 (2006)

Norstrom, M.: Geographic information system(GIS) as a tool in surveillance and monitoring of animal disease. Acta veterinaria Scandinavica Supplement 94, 79-85 (2001)

Xiangyan, T., Baoxu, H., Xueguang, Z.: The application of GIS in animal health domain. Journal of Chinese veterinarian 41(6), 58-60 (2005)

Pferiffer, D.U., Hugh-Jones, M.J.: Geographical information systems as a tool in epidemiologicl assessment and wildlife disease management. Rev. Sci. Tech. Off. Int. Epiz 21(1), 91-102 (2002)

Greenough, G., McGeehin, M., Bernard, S.M.: The potential impacts of climate variability and change on health impacts of extreme weather events in the United States. Environ. Health Perspect. 109(suppl. 2), 191-198 (2001) 